and 75 adults from the war region. Researchers administered the Childhood Post-traumatic Reaction Index, anxiety questionnaires, projective methods, and clinical case examples. Observed reactions in the three groups of subjects (pre-school, school-age, adolescents) are as follows: (1) phobic reactions; (2) psychosomatic disturbances; and (3) affective disorders. The most frequently observed defense mechanisms found were denial, regression, splitting, altruism, and sublimation. Examples of each will be presented with clinical material.

A key issue in the psychological response to disaster is the effect of bereavement and loss. Individual reactions are determined by one's developmental stage, temperament, past experience with trauma, and resiliency. The powerful link between response to trauma and social cultural factors are elaborated.

1. The JFDP is an academic exchange program administered by the American Councils for International Education (ACTR/ACCELS), and is fully funded by the Burcau of Educational and Cultural Affairs of the United States Department of State.

Keywords: children; coping; defense mechanism; disasters; psychological response; traumatic events

Prehosp Disust Med 2005;20(2): $\mathrm{s} 2-\mathrm{s} 3$

Culture-Sensitive Aspects of Psychosocial PostDisaster Care in the Netherlands after the Bijlmermeer Airplane Crash and the Enschede Fireworks Disaster J. Netten

Impact Foundation, Netherlands

Introduction: When a disaster strikes, a large group of people may be affected either mentally or physically. This group, although united by their communal ordeal, may consist of people of different ethnic or cultural origin. Ethnic or cultural minorities generally are considered to be at-risk groups when it comes to the effects of a disaster. In 2003, Impact, the Dutch knowledge center for post-disaster psychosocial care, started a project called "Lessons Learned". This project reviews the psychosocial interventions in the Netherlands regarding the affected ethnic minority groups of the Bijlmermeer airplane crash (1992) and the Enschede fireworks disaster (2000) in order to make recommendations for psychosocial interventions if disasters occur again.

Methods: Obstacles are identified and recommendations are formulated after analyzing $>55$ evaluation reports, scientific studies on psychosocial interventions after the two disasters, descriptions of treatments, and by interviewing various caretakers who were involved with the preventive, curative, and care interventions.

Results: Language problems emerged quickly, causing communication gaps during the aftermath of the disasters. To improve communication, a number of measures were taken from the start, and others were added after some time. Cultural differences and codes interfered with reaching the various ethnic groups, and with the success or failure of the available treatments. Some other factors were important as well. A multidisciplinary approach, cooperation between (mental) health institutions and community centers, and the sharing of knowledge and information all were essential in mitigating the psychosocial consequences of a disaster for everyone concerned. A continuous update of skills and knowledge for (mental) health personnel regarding a multicultural population remains an issue that should not be overlooked.

Conclusion: The Dutch experience with two major disasters makes it clear that a uniform psychosocial approach may not be sufficient to provide psychosocial care to all affected people. When it comes to preventive actions, interventions during the acute phase and psychosocial aftercare on the medium- and long-term and a more finetuned approach geared to the special needs and conditions of various sub-groups is essential to help alleviate the pain and stress caused by a disaster.

Keywords: care; communication; cooperation; cultures; language;

Netherlands; post-disaster; psychosocial

Prehosp Disast Med 2005:20(2):s3

\section{Humor and Religion: How an Emergency Department Coped with the 2003 Severe Acute Respiratory Syndrome (SARS) Outbreak}

K.Y.Tham

Tan Tock Seng Hospital, Singapore

Objective: This study examined the psychological coping strategies adopted by emergency department (ED) healthcare workers who cared for Severe Acute Respiratory Syndrome (SARS) patients during the outbreak in Southeast Asia in the spring of 2003.

Methods: During the outbreak from 13 March to 31 May 2003 , the ED in the study was Singapore's only SARSscreening center and was closed to all other patients. The use of personal protective equipment and directives for infection control were strictly enforced. Heathcare workers experienced unusual stressors, namely, the unknown nature of the disease; the fear of infection, contagion, and death; stigmatization and discrimination; disruption of normal work and lifestyle; and conflicts with the sense of duty to care for patients and sick colleagues. To help healthcare workers cope, the study hospital and ED introduced psychosocial measures including: (1) enhanced communication within the hospital and ED; (2) enhanced communication with the community and public relations management; and (3) welfare and psychological support for healthcare workers.

In November 2003, a self-administered survey of ED doctors and nurses was conducted. Data collected included demographics and responses to Coping Orientation to Problems Experienced (COPE), which groups coping responses, according to 15 scales, each with a minimum score of 4 and maximum of 16 . The higher score in a scale meant more use of that coping response. The scales were then categorized into problem-focused and emotionfocused strategies, which were adaptive, or lessuseful/adaptive strategies.

Results: Thirty-eight (92.7\%) of 41 doctors and 58 (69.9\%) of 83 nurses responded. The mean age of the doctors was 31.6 years old (standard deviation $(\mathrm{SD})=4.4$ ) and the mean age of the nurses was 32.1 years $(\mathrm{SD}=9.2)$. Respondents scored 9.9/16 (95\% Confidence Interval $(\mathrm{CI})=9.5-10.3)$ 